Proceedings of SALT 26: 1022-1042, 2016

\title{
Shapes of conversation and at-issue content *
}

\author{
Julie Hunter \\ GLiF, Universitat Pompeu Fabra \\ Nicholas Asher \\ IRIT, CNRS
}

\begin{abstract}
While not-at-issue (NAI) content is typically introduced via particular syntactic constructions, accounts of NAI content that only consider the syntax/semantics interface face empirical difficulties. We develop a new approach to NAI content that exploits features of discourse structure to solve these difficulties, and we provide some insight on the strategic uses of NAI constructions.
\end{abstract}

Keywords: discourse structure, appositives, not-at-issue and at-issue content

\section{Introduction}

A recurrent theme in discussions of at-issue (AI) and not-at-issue (NAI) content is that "a single constituent (which we take to be a linguistic clause) can simultaneously contribute multiple independent meanings" (Potts 2012). Discourse theories like Segmented Discourse Representation Theory (SDRT; Asher 1993; Asher \& Lascarides 2003) propose a similar idea: a single linguistic clause can introduce several, distinct discourse units (DUs), each of which may have one or more distinct discursive roles in the overall structure of the discourse. We show that conventional implicatures generated by appositives as well as other NAI constructions have a compelling analysis when we substitute discourse units for multiple independent meanings in Potts' quote. Our proposal has three parts. First, we argue that extant accounts that have NAI and AI content contribute to distinct dimensions of content or be evaluated relative to distinct update mechanisms are ultimately untenable. Second, using SDRT, an established theory of discourse structure, we show how to provide a discourse based account of the phenomena that have motivated the AI/NAI distinction, and we argue that a simple division of content into NAI/AI categories based on rejection continuations (No, that's not true) should be replaced by a more nuanced view that considers many other types of continuations. The resulting account appeals to a single dimension of semantic content and a uniform mechanism for update, but allows that discourse updates can be structured in a way

* We thank ERC research grant 269427 for research support. Thanks to Robert Henderson, Katja Jasinskaja, Sophia Malamud, Eric McCready, Paul Portner, Chris Potts, Malte Willer, and five anonymous SALT reviewers for helpful comments on the account developed in this paper. 
that influences the acceptability of future discourse moves. Finally, we show how conversational goals and strategies explain why NAI expressions are useful rhetorical devices. ${ }^{1}$ Using a game-based theory of conversational strategies and goals (Asher, Paul \& Venant 2016; Asher \& Paul 2013), we model the strategic contribution of AI content and NAI/AI shifts over many conversational turns.

\section{Background on appositives and NAI constructions}

Wilson (1975) described what was already a known peculiarity about certain types of expressions: that the contributions of these expressions do not fall within the scopes of modal operators. The types of expressions that fall into this category are rather varied; they include mood indicators for questions and commands, interjections like oh, gee, too bad, damn, discourse adverbials like allegedly and unfortunately, adverbial clauses like Mary assures us, so called pragmatic conditionals like if you know what I mean or if you see what I'm getting at, and discourse particles like tte in Japanese or $r e$ in Sissala for marking hearsay evidence, parenthetical constructions and discourse connectors like but, too, hence, so, and therefore. To this list Asher (2000) added non-restrictive relative clauses and appositive NPs. The list bears a significant similarity to the list of what current semanticists and pragmaticists take to be NAI constructions, though presupposition triggers are missing. What Wilson called a failure to pass the embedding test, linguists now call projectability.

Wilson concluded from her embedding test that expressions that convey NAI content do not convey truth-conditional content. But as Asher points out, her conclusion concerning non-truth-conditional meaning ignores the possibility that the content of the apparently non-truth-conditional item may fall outside the scope of the conditional but nevertheless contribute to the truth conditions of the discourse.

Recent proposals along the syntax-semantics interface have sought to capture the semantic contribution of NAI content while explaining its unique properties. These accounts echo Wilson's description in that they separate the semantic contribution of AI content from that of NAI content in an effort to capture the "scopelessness" of NAI content. Unlike Asher, however, they attempt to model the semantic contribution of both AI and NAI content without appealing to discourse structure. It's easy to see why: constructions that have been classified as NAI constructions make very effective tools for backgrounding the information they convey, in the sense of making it less central to the main themes of a discourse and indicating that a speaker takes this content for granted and does not see it as up for discussion or "at-issue". One need only make the assumption that the ability of such a construction to serve as a tool for conveying NAI content arises from the nature of the construction itself;

1 In this we sympathize with Amaral, Roberts \& Smith (2007) and Roberts (2012) though we develop the idea differently. 
just as the semantic function of a negation marker such as not is to negate things, the semantic function of so-called NAI constructions is to make things not at-issue. Thus we might expect these constructions to convey NAI content whenever they are used, regardless of the surrounding discourse structure. ${ }^{2}$

To capture the behavior of NAI constructions, Potts (2005) posits that NAI and AI constructions contribute their contents to separate dimensions of semantic content. In particular, Potts focuses on the contribution of the expressions that he classifies as conventional implicatures, including supplemental clauses such as as-parentheticals, non-restrictive (or appositive) relative clauses, and nominal appositives. ${ }^{3}$ The multidimensional logic that Potts develops models many important features of CI contents: it allows them to make a contribution to the semantic content of the sentence in which they figure while remaining "logically and compositionally independent" of AI content, which permits them to escape the scope of embedding operators.

Arguably, however, the account separates CI and AI content - and NAI and AI content more generally - too well: it appears to predict that any semantic interaction between NAI and AI content is impossible, yet as AnderBois, Brasoveanu \& Henderson (2015) show, there is abundant evidence of presuppositional, anaphoric and elliptical links between NAI and AI contents. ${ }^{4}$ Interestingly, these dependencies seem to run in both directions:

(1) John, who nearly killed a woman with his car, visited her in the hospital. (AnderBois et al. 2015)

In (1), the pronoun his appears in an appositive relative clause and is therefore CI content (so NAI), but it is linked to John, which is taken to be AI. The link between her and a woman illustrates that AI content can likewise depend on CI content.

To capture the observed anaphoric interactions between AI and NAI content, AnderBois et al. develop a mono-dimensional, dynamic account in which CI and AI content is evaluated relative to two different dynamic update mechanisms, and presupposed content is treated as a precondition on the Common Ground. In particular, AnderBois et al. propose that AI content be modelled as a proposal to update a set of common grounds (CG): a proposal, if accepted, eliminates from each element of CG those worlds that do not satisfy the AI content (cf. Farkas \& Bruce 2010). On the other hand, CI content eliminates elements from CG, i.e. sets of worlds,

2 Note that providing a model of NAI content along the syntactic-semantic interface does not preclude invoking a discourse-level explanation for when and why we employ NAI constructions. For example, in section 4 of their paper, AnderBois et al. (2015) apply their (non-discursive) model of NAI content to explain certain discourse-level behaviors exhibited by NAI and AI contents.

3 Potts also includes supplemental adverbs and expressives, but we focus here on supplemental clauses, and non-restrictive relative clauses in particular.

4 See also Amaral et al. (2007). 
that do not satisfy the CI content. Since what is up for discussion are only those propositions whose status is not fixed in a candidate CG and as the update for CI content automatically updates the CG, CI content is not up for discussion, and not at issue. To permit anaphoric connections between CI and AI content, AnderBois et al. adopt a dynamic update procedure that allows AI and NAI content to be updated incrementally. Update of a sentence such as (1) proceeds so that John first contributes a proposal to update the CG with a discourse referent identified with John, then the appositive content is automatically imposed on the CG in the way described above, and then the remainder of the main clause content is treated as a proposal to update this recently changed CG. The salient features of this account are that on the one hand, update is incremental in order to support anaphoric connections between CI and AI content; but on the other hand, appositive content is added to the CG (because it is automatically imposed) before the main clause content is added, making the appositive immune to the influence of logical operators in AI content.

The two distinct mechanisms involved in the update of (1), however, are interdependent, and so what's NAI may be more than what is in the CI expression. AnderBois et al. say that presuppositional dependence of NAI content on AI content forces update of the latter on the CG; about (2), they say that the presupposition triggered by too, which is notoriously difficult to accommodate, requires that the content of John kissed Mary be added to the CG before the appositive can be interpreted.

\section{John kissed Mary, who kissed HIM TOO}

However, the antecedents of pronouns (including relative pronouns) are also notoriously hard to accommodate, so their antecedents, e.g. the discourse referent introduced by John in (1), should also be added to the CG prior to the update with appositives that depend on them. Although the update mechanism for John is different from the update mechanism for CI content, it's effect is the same: the contribution of John in (1) can no longer be AI if the CI content which depends on it is NAI. ${ }^{5}$

An empirical problem arises if we tie distinct NAI and AI update mechanisms to syntactic constructions like supplemental clauses regardless of their position in the text. Sentence-final appositives appear to behave very much like AI contents (as AnderBois et al. acknowledge). ${ }^{6}$

5 Another reason to assume that the contribution of John is not actually AI in (1) is that proper names are arguably presuppositional and are generally treated as such in dynamic frameworks. In fact, in all of the examples that AnderBois et al. provide with sentence-medial appositives - that is, sentences of the form AI + NAI + AI - the first AI contribution (e.g. John) involves an arguably presuppositional expression.

6 See Syrett \& Koev 2015 for experimental evidence of the difference between sentence-medial and sentence-final appositives with regard to discourse continuations. 
(3) In springtime, the fish swim upriver and give birth to live young, who then spend their first months living on land. (variant on AnderBois et al.'s (92)).

(4) While she was filming Fresh Prince, her mother, who then worked as a librarian, would call her with Black history stories. (variant on AnderBois et al.'s (93))

AnderBois et al., like Potts (2005), assume that supplemental clauses always contribute CI content. Yet in (3), the content of the appositive appears to be very much up for discussion. For example, an utterance of "No, that's not true!" would easily be taken to target the content of the appositive. By contrast, it is highly unlikely that such a response to (4) would be interpreted as targeting the appositive. Sentence-final appositives seem to stay open for discussion in a way that medial appositives do not.

In an effort to explain away the threat that (3) and (4) pose to their account, AnderBois et al. suggest that then moves the reference time forward in final appositives, while it retains the reference time of the main clause in medial appositives. They use this distinction, with observations about continuations like "No, that's not true!", to motivate an ambiguous treatment of medial and final appositives. Yet an appositive's syntactic position does not determine the interpretation of adverbials like then. The appositive in (4) can have the same interpretation when sentence final:

(4') While she was filming Fresh Prince, she would receive a new book each week from her mother, who then worked as a librarian.

It seems rather that the placement of the appositive affects its NAI status, counter to the assumption that appositives always contribute NAI content.

A final and fatal difficulty with the idea that the AI/NAI distinction rests on a distinction between proposals to update and automatic impositions or constraints on the CG is that it gives rise to an absurd consequence: just by using a particular syntactic structure, a speaker can force her interlocutor to commit to a particular content. Not only is this highly unintuitive, but it's demonstrably untrue for examples like (5), taken from the Princess Bride:

(5) Buttercup: (a) He [Humperdink]... can find a falcon on a cloudy day, he can find you!

Wesley: (b) You think your dearest love will save you?

Buttercup: (c) I never said he was my dearest love, and yes, he will save me. That I know.

Wesley: (d) You admit to me that you do not love your fiancé?

Buttercup: (e) He knows I do not love him.

As background, Buttercup became engaged to Prince Humperdink, whom she does not love, after she came to believe that her true love, Wesley, had been killed by 
pirates. In this scene, she has been taken hostage by a pirate. She believes him to be the pirate who killed Wesley, but unbeknownst to her, he is Wesley in disguise.

In (5a), Buttercup is trying to convince Wesley to release her by telling him that she will be saved whatever he does. Wesley follows up in (5b) with a seemingly on-topic confirmation question concerning Buttercup's goal. However, using a presuppositional construction, ${ }^{7}$ which gives rise to NAI content, he sneaks in a question of his own: whether Buttercup loves Humperdink. He follows up on this question in (5d) and continues to return to the topic in later scenes. In fact, we come to realize that Wesley disguised himself in the first place precisely because he wanted to get an honest answer to this question. If we assume that Buttercup is automatically committed to the content of the presupposition, however, we lose sight of the fact that Wesley is looking to see whether she accepts this content or not and the fact that her letting the content pass without correction would be incriminating - that is, she would be the one held accountable for her commitment to this content, not him.

One might respond that we can re-describe this situation in a way that is compatible with AnderBois et al.'s account: Wesley uses a presupposition to automatically update Buttercup's commitments (through automatic accommodation in the CG) and then tests her to see whether she will revise this update or not. We see multiple problems with this description. First, it is unintuitive: Buttercup rejects the content, and there is no reason to think that she was committed to it prior to her objection. Second, AnderBois et al. do not provide an account of correction of CG content. Third, we do not see how they could develop such an account without collapsing the two update mechanisms. The CG has been automatically updated with the presupposition that Humperdink is Buttercup's true love, so there are no worlds left in the $\mathrm{CG}$ in which this content is not true. To make sense of the correction, worlds in which Buttercup does not love Humperdink will have to be (somehow) reintroduced, and the proposal that she loves him will have to be put on the table. That is, the presupposed content will have to be treated as AI content. In our account, which we develop in the next section, utterance content does not disappear into the CG when the discourse moves on. Representational discourse structures track and store the content of each discourse unit, allowing the content of past utterances to be targeted by correction, which we model as a discourse relation.

7 We could rework this example using an appositive, e.g.: W: You think that Humperdink, whom you love so dearly, will save you? B: I never said that I loved him, and yes, he will save me.... Using the original presupposition, however, is powerful because if it is ever reasonable to assume that an interlocutor can employ an NAI construction to force his interlocutor to commit to its content, it seems that presuppositional constructions would make the best candidates. 


\section{A discourse account}

The undesirable consequences of Potts 2005 and AnderBois et al. 2015 stem from the assumption that the NAI status of content conveyed by what have been classified as NAI devices is a direct consequence of their syntactic and semantic features. In this section, we show that the NAI status of certain constructions follows from general principles of discourse structure. The account that we develop allows semantic interactions between AI and NAI content and accounts for the data on sentence-final appositives, and it does so without requiring automatic impositions of content on the CG. In fact, our account makes no assumptions about the CG whatsoever. The CG is irrelevant to our account.

We start with the idea that a speaker cannot force an interlocutor to commit to content, but that it is within the power of a speaker to construct her discourse in such a way as to make certain content more or less accessible for discourse continuations. Compare (6) and (7).

(6) A guy at work, who has a sister in fashion design, gave me this shirt.

a. ? She works for Stella McCartney.

b. I like it a lot.

(7) I got this shirt from a guy at work, who has a sister in fashion design.

a. She works for Stella McCartney.

b. I like it a lot.

While judgments on discourse continuations and the role of the appositives in (6) and (7) are subtle, it seems clear that one cannot build off of the appositive in (6) in a way that completely ignores the content of the main clause by ignoring the guy at work and the shirt that he gave the speaker. ${ }^{8}$ To the extent that (6a) is an acceptable continuation of (6), we need to understand a relation between Stella McCartney and the shirt that was given - a relation not provided by the example as it stands.

In the rest of this section, we lay out an account of NAI and AI content that is driven mainly by data on discourse continuations that are not limited to corrections or rejections of content. In our account, the idea of a binary distinction of types of content dissolves in favor of a more complex and dynamic picture of information that is accessible to continuations of different types to different degrees.

8 The interpretation of appositives is also sensitive to the preceding discourse, when the sentences in which they figure are not discourse initial. As many, including AnderBois et al. (2015), have observed, it is very difficult, if not impossible to construct an example in which the appositive is relevant to the preceding discourse while the main clause is irrelevant to it. 
Shapes of conversation

\subsection{Discourse structure}

Discourse theories start from the undeniable intuition that discourses have an organization beyond the level of a sentence and that each clause, or relevant unit of content, plays certain semantically and pragmatically important roles relative to other units of content, so as to form a relational structure that represents the content of the whole discourse. Linguistic theories of discourse structure like SDRT characterize these discourse structures and their semantic and pragmatic effects.

Characterizing a discourse structure involves solving three problems. First, how do we individuate the elementary discourse units (EDUs) of discourse content that play semantically and pragmatically important roles? Second, what types of relations hold between EDUs? Third, what are the constraints, if any, on how a discourse can be structured? All three tasks are relevant to the concerns of this paper. Starting with the first, if in a given sentence, some content projects out from operators (i.e., it fails the embedding test), then the expressions determining that content should form at least one EDU. Another sufficient (and non-necessary) condition for EDU segmentation in SDRT is that every clause contribute at least one EDU to the discourse. As for the second problem, we assume with SDRT that discourse units are related by various rhetorical relations, including Question Answer Pair (QAP), in which one discourse unit answers a prior unit characterized by a question, and Explanation, in which one discourse unit provides an explanation for the eventuality described by another. We address the third task below.

Our vocabulary for describing discourse structures contains a countable set of discourse unit labels $D U=\left\{\pi, \pi_{1}, \pi_{2}, \ldots\right\}$ and a finite set of discourse relation symbols $R e l=\left\{R, R_{1}, \ldots R_{n}\right\}$, which we add to the vocabulary of a language $L$ for describing EDU contents (e.g., the language of dynamic predicate logic). Formulas in the SDRT language are of the form $\pi: \varphi$, where $\varphi$, which describes the content of $\pi$, is: a formula of $L$; a formula of the form $R\left(\pi_{1}, \pi_{2}\right)$, which says that $\pi_{2}$ stands in coherence relation $R$ to $\pi_{1}$; or a conjunction of SDRT formulas. Following Asher $\&$ Lascarides (2003), each discourse relation comes with constraints as to when it can be coherently used in context and when it cannot. ${ }^{9}$ We note that in SDRT several discourse units can be grouped together as a complex discourse unit (CDU) that can be characterized via an SDRT formula. A CDU is formed when a group of DUs (EDUs or CDUs) work together to form an argument to a rhetorical relation.

A discourse structure for a text can be represented as a graph $\left(V, E_{1}, E_{2}\right.$, Last,$\left.\ell\right)$, where $V \subseteq D U$ is a set of vertices each representing a discourse unit; $E_{1} \subseteq V^{2}$ a

9 While discourse structure inferences are typically nonmonotonic or probabilistic, implemented SDRT models (Muller, Afantenos, Denis \& Asher 2012; Afantenos, Kow, Asher \& Perret 2015; Perret, Afantenos, Asher \& Morey 2016) have proven predictive over large corpora of discourse annotated, extended texts. 
set of directed edges representing links between discourse units that are labelled by $\ell: E_{1} \rightarrow R e l$ with discourse relations; $E_{2} \subseteq V^{2}$ describes the membership relation between the set of DUs figuring in CDUs and the CDUs in which they figure; and Last $\in V$ is the last EDU in the linear, textual ordering of EDUs in $d$. An SDRS is spanning in that all elements of $V$ other than the root have at least (and possibly more than) one incoming edge: $\forall \pi_{x} \in V .\left(\pi_{x} \neq\right.$ ROOT $\rightarrow \exists \pi_{v} \in V$. $\left.\left(\left(\pi_{v}, \pi_{x}\right) \in E_{1}\right)\right)$.

We return now to the task of determining constraints on discourse structures. The most relevant for our current purposes is the Right Frontier Constraint (RFC), which determines how discourse units can attach to a contextually given discourse structure. The RFC requires that given a discourse graph $G$, a new EDU to be attached to $G$ must be attached to a node along the Right Frontier (RF) of $G$. Nodes that are not on the RF can be accessed, but only through what Asher (1993) calls discourse subordination. This occurs when a speaker explicitly brings content back into salience, e.g. by saying, Going back to your first point about the economy... after the conversation has moved on to another point. To define the RF of a graph $G$, SDRT distinguishes two types of edges in the set $E_{1}$, coordinating and subordinating. Relations such as Explanation, Elaboration, and Background - in which the second argument seems to add information about the first, rather than push the discourse forward-are represented with subordinating (vertical) edges. Relations such as Continuation, Narration, and Result - in which the second argument shuts off the accessibility of the first - are represented with coordinating (horizontal) edges.

Let $e\left(\pi_{x}, \pi_{y}\right)$ mean that edge $e$ has initial point $\pi_{x}$ and endpoint $\pi_{y}$. A node $\pi_{x}$ is on the RF of a graph $G$, i.e. $\mathrm{RF}_{G}\left(\pi_{x}\right)$, just in case $\pi_{x}$ is Last, $\pi_{x}$ is related to Last via a series of subordinating $(\mathrm{Sub})$ edges, or $\pi_{x}$ is a CDU that includes a node in $\mathrm{RF}_{G}$ :

Definition 1 Let $G=\left(V, E_{1}, E_{2}\right.$, Last,$\left.\ell\right)$ be a discourse graph. $\forall \pi_{x}, \pi_{y}, \pi_{z} \in V$, $\mathrm{RF}_{G}\left(\pi_{x}\right)$ iff $(\boldsymbol{i}) \pi_{x}=$ Last, (ii) $\mathrm{RF}_{G}\left(\pi_{y}\right) \& \exists e \in E_{1}, e\left(\pi_{x}, \pi_{y}\right) \& \operatorname{Sub}(e)$, or (iii) $\operatorname{RF}_{G}\left(\pi_{y}\right)$ $\& \exists e \in E_{2}, e\left(\pi_{x}, \pi_{y}\right)$.

Note that the RF is updated dynamically each time a new EDU is processed; the RF for (attachment of) an EDU $\pi_{n}$ will be determined by the graph $G_{\pi_{0}-\pi_{n-1}}$. The RF for a CDU $\pi_{m} \ldots \pi_{n}, m<n$, is the RF for $\pi_{m}$.

\subsection{Appositives revisited}

The discourse structures characterized above allow us to explain many important features of appositives. First, an appositive will contribute an EDU distinct from that contributed by its main clause, which means that it need not remain in the scope of an operator that has scope over the main clause; whether it does or not depends on the

attachment heuristics for appositives. Second, we distinguish appositives (without presupposition triggers) from presuppositions via the relations that attach these 
constituents to the discourse context: presuppositions can be bound via a particular discourse relation (Consequence), whereas CIs, like all discourse-new content, must attach via discourse relations other than Consequence (Asher \& Lascarides 1998). And SDRT's dynamic semantics models anaphoric connections between CI and AI content: when certain structural constraints, including the RFC, are met, anaphoric links can hold between expressions figuring in mutually accessible DUs (Asher \& Lascarides 2003).

SDRT predicts the observation from AnderBois et al. 2015 that sentence-final appositives license continuations more easily than medial appositives do. Sentencefinal appositives will be on the RF, because the appositive will be Last, and will therefore remain accessible for discourse continuations. By contrast, when the appositive is medial, the main clause will be Last because it will be the last complete DU added to the discourse, and the appositive will be inaccessible because of the way it attaches to the main clause.

In more detail, let us revisit examples (3) and (4).

(3) $\quad$ In springtime, $]_{\pi_{1}}$ [the fish swim upriver and give birth to live young $]_{\pi_{2}}$, [who then spend their first months living on land. $]_{\pi_{3}}$

(4) [While she was filming Fresh Prince, $]_{\pi_{4}}$ [her mother, [who then worked as a librarian, $]_{\pi_{5}}$ would call her with Black history stories. $]_{6}$

First, we note that in (3), the appositive is Last: [main clause $]_{\pi_{n-1}}[\text { appos }]_{n}$, while in (4) the main clause is Last: [main [appos $]_{\pi_{n-1}}$ clause $]_{\pi_{n}}$. Next, the units in (3) and (4) are related with different rhetorical relations (cf. Asher \& Lascarides 2003).

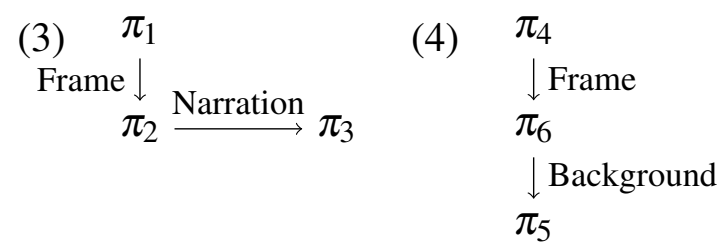

In (3), attachment of $\pi_{3}$ to $\pi_{2}$ renders $\pi_{2}$ (the main clause) inaccessible, but puts $\pi_{3}$ on the RF. We therefore predict that building off of the appositive in (3) should be easy, while building off of the main clause in a way that ignores the appositive, is difficult. This prediction is illustrated in the contrast between the following continuations to (3): ... ? They die shortly thereafter.

(9) ... Using their fins as "feet", they are able to move through the grass to find insects to eat.

In (4), $\pi_{6}$ is Last and $\pi_{4}$ is super-ordinate to it, meaning that both $\pi_{6}$ and $\pi_{4}$ are 
on the RF. $\pi_{5}$, however, is subordinate rather than super-ordinate to Last/ $\pi_{6}$ and is therefore off of the RF and inaccessible (cf. the infelicity of (6a)). ${ }^{10}$

The principles of SDRT dictate that a medial appositive cannot serve as the second argument to a coordinating relation that attaches to Last (Asher 2000; Asher \& Lascarides 1998). To see this, consider AnderBois et al.'s (2015) infelicitous (10).

?? John kissed Mary, who then kissed him, at the concert in the park.

Let $\pi_{1}$ be the appositive and let $\pi_{2}$ be the main clause. Our account predicts that $\pi_{2}$ is Last and therefore on the RF. If we attempt to construct a discourse structure for (10), however, we get the formula Narration $\left(\pi_{2}, \pi_{1}\right)$. Since Narration is coordinating, this entails that $\pi_{2}$ is not on the RF, contrary to what its status as Last entails. This structure is unacceptable, and so (10) is predicted to be infelicitous. By contrast, a medial appositive is free to serve as the second argument to a subordinating relation, because this does not threaten the accessibility of the main clause.

An SDRT-based account makes further predictions that appear to be borne out. First, it predicts that the main clause in (4) and (4') (in which the appositive is sentence-final) will be on the RF and thus accessible regardless of the position of the appositive (cf. (6b) and (7b)).

(11) While she was filming Fresh Prince, her mother, who then worked as a librarian, would call her with Black history stories. Many of them moved her to tears/She spent hours on the phone.

(12) While she was filming Fresh Prince, she would receive a new book each week from her mother, who then worked as a librarian. Many of them moved her to tears/She spent hours reading them.

Second, our account predicts that discourse continuations can pick up on the content of a medial appositive so long as the appositive figures in a CDU that is on the RF. Consider the following examples:

(13) a. Marie, the best volleyball player in the district, is joining our team. We're going to be invincible!

b. Marie, the worst volleyball player in the district, is joining our team. ? We're going to be invincible!

The continuation (We're going to be invincible!) is sensitive to the content of the appositive-(13b) can only have an ironic reading. In this case, the appositive and main clause function together to justify the continuation.

Interestingly, our account leaves the door open for discourses like (14).

10 See Jasinskaja 2016 for a similar but independently developed proposal. 
Shapes of conversation

(14) [The candidate must submit her manuscript by Dec. 1 to the committee, $]_{\pi_{1}}$ [who must then approve it within 5 days, $]_{\pi_{2}}$ [in order for the candidate to graduate on time.] $\pi_{3}$.

In (14), $\pi_{1}$ is relevant to the discourse continuation $\pi_{3}$ despite its being related to $\pi_{2}$ via Narration. This is possible for two reasons. First, even though we may have a single syntactic constituent at the level of IP, we have three separate discourse constituents with the medial appositive as the second constituent. This gets around the problem posed by (10). Second, much like the appositive and main clause in (13a), $\pi_{1}$ and $\pi_{2}$ in (14) work together as a CDU to support the continuation with $\pi_{3}$.

\subsection{A fresh look at not-at-issue content}

The account that we have developed above differs from alternative accounts in various ways. While we agree that NAI constructions are good tools for forcing their content to play a secondary role in the discourse, we reject the claim from models along the syntax-semantics interface that the syntactic and semantic features of these constructions are solely responsible for their ability to do so. In our account, syntactic and semantic features are crucial for determining the discourse contribution of a given sentence - they help to determine, for example, what EDUs are contributed to a discourse and also how these EDUs are attached to one another. However, whether or not the configuration of discourse units contributed by a sentence containing an NAIdevice allows the NAI content to interact with discourse units prior or subsequent to the main clause follows from general principles of discourse structure.

In emphasizing the role of discourse in the interpretation of NAI constructions, our account echoes sentiments in Amaral et al. 2007 and Simons, Tonhauser, Beaver $\&$ Roberts 2010. The model that we offer is, however, importantly different. Not only is it not a QUD-based model, but we do not assume that the discursive roles of discourse segments reflect in any overt way the conversational goals of interlocutors. We discuss the relation between goals and discourse structure in Section 4.

Another important way in which our account differs from the other accounts that we have considered concerns the role of commitments in our model. We, like AnderBois et al. (2015), find the proposal-based account of assertions from Farkas \& Bruce 2010 very insightful. At the same time, we think that one should be careful in extending this model to the contents of sentences that convey multiple kinds of meaning. The motivation to model NAI content as imposing, à la AnderBois et al. $2015,{ }^{11}$ an automatic update of the CG seems to be the following. The felicity of no (or yes) as a response to an assertion motivates treating the content of that assertion as a proposal; when uttered as a response to a sentence containing an appositive, no

11 See also Murray 2011 for a closely related approach to evidentials. 
does not target the appositive (leaving sentence-final appositives aside); therefore, appositives should not be analyzed as proposals to update the CG. This reasoning discounts the possibility that a speaker might put forward a multi-part proposal, which would require her interlocutor to adopt different strategies for rejecting the different parts.

In fact, while no/yes continuations provide a strong motivation for treating simple assertions as proposals, these interactions do not yield a model of correction of individual discourse units. Take, for example, (15):
A: John made the cake.
B: No, it was Mary.

A simple no would have a different reading from B's response. A rejection can target even the content of a single segment in different ways. In SDRT, rejections or corrections with no involve a structural relation. Structural relations, including Correction and also Contrast, sometimes focus on just a particular part of a constituent, whether simple or complex, that they take as an argument. As detailed in Asher \& Lascarides 2003, B's correcting move in (15), if it just stops with no is rather odd; we know that B objects to what A said but not exactly what she finds objectionable. The continuation it was Mary makes precise what B objects to: it wasn't that there wasn't a cake made, it was just that it was Mary who made it.

When we scale up to larger discourses, we again need to clearly circumscribe the target of a rejection, either by explicitly stating it, as in (18), or by relying on features of the discourse itself, as in (16). ${ }^{12}$ Consider the following examples, found through Google:

(16) "You did not know that because your identity was transmitted TBF-To Be Forgotten-in order to mask you living in the world with humans. You were never to know the truth...Y You had the chance to be normal like them, Sarabel. But you've lost it now," she said, "I'm sorry". A fury of emotion pillaged

12 In this paper, we will not explore the features that guide how a rejection will be understood. We observe nevertheless that features of the larger discourse can play a role. In (16), the vast majority of the content being rejected (much of which is left out in the excerpt above) is dedicated to telling Sarabel that she is an alien; I'm sorry is merely a comment on this larger topic. In (17), the interpretation depends on whether we understand all of Abby's comment as forming a CDU. We also note that the kind of discourse relation might make a difference. Suppose I say, John is going to be late because his bike is broken and someone corrects me with no, that's not true. If we take the correction as targeting the content his bike is broken, then where does that leave my claim that John is going to be late? It's hard to reject one part of an Explanation without calling its other part into question. Finally, there are rules about dialogue that are relevant to the interpretation of correction but which go beyond discourse structure as we have presented it here. For instance, the interpretation of a correction will depend in part on when interlocutors are allowed to reject. 
Shapes of conversation

through Sarabel then. Tears fluttered through her eyelashes. "No! That's not true! It's just not true! What about my parents?" Sarabel asked. "They are one with us as well. But you mustn't tell them that. Let them live the life you can no longer have," she said. "No!" Sarabel shouted, "It isn't true!".

Abby: "Dad said you cheated on him, that you wanted a divorce, you met another man." Jill "No, that's not true".

(18) R.S.: "No, they're not pushable. They are sellouts. They are co-opted. They-"

T.O.: "That's not true. She's clearly pushable."

Examples (16)-(18) bring out another important point, namely that corrections like No, that's not true need not target the most recent utterance in a discourse. Koev (2012) emphasizes, as we have, the influence of the sentential position of an appositive in its interpretation. However, Koev arrives at the conclusion that recency is the most important feature in determining whether something is "at-issue" or not, and thus whether it will be targeted with a correction such as No, that isn't true. (16)-(18) refute this claim; in (17), for example, the rejection can target the CDU formed from Abby's entire comment. In fact, many of the examples discussed in our presentation of our discourse approach undermine this claim because they show that the main clause and the appositive can both be accessible so long as the appositive is attached via a subordinating relation. It is precisely due to attachment data like these that discourse theories tend to treat Last as a special unit while also laying out rules for when a discourse unit can attach to a unit other than Last.

Still, we agree with Koev (2012) and Amaral et al. (2007) that examples with medial appositives interact differently with corrections than their variants without appositives. Consider (19), a variant of an example from Amaral et al. 2007.
a. Edna is always very brave, so they let her start the descent.
b. They let Edna, who is always very brave, start the descent.
c. No, that's not true/she isn't. She is a coward.

A highly context dependent correction like No, that's not true must target an element on the RF. The fact that (c) can target the first sentence of (a) shows that in dialogue, an interlocutor $i$ can ignore the speaker's last utterance, even if it pushes the RF of the speaker's representation forward, if $i$ hasn't been given a chance to correct the preceding utterance; an interlocutor commits to the content of a discourse graph only by explicitly building on it or by passing up a chance to correct it. Ignoring the content following the appositive in (b), however, would lead to an anomalous result: $i$ would commit to an EDU with the fragmentary content, they let Edna. 
SDRT does allow, however, for discourse subordination (Asher 1993), in which elements off the RF can be made available by the use of complex descriptions or the repetition of relevant content. We saw an illustration of this in (5c), but we also observe that She is a coward alone would be an acceptable correction of (19b). Prosody also has important effects on discourse structure (Reese 2007). A final falling intonation contour on the medial appositive might affect its position in the overall discourse structure, making it accessible according to our definition of the $\mathrm{RF}$, as might a particular contour on the continuation. ${ }^{13}$ We leave the effects of prosody on the status of (N)AI content for future research.

The idea of $(\mathrm{N}) \mathrm{AI}$ content that emerges from our view undermines the binary distinction proposed in other accounts in favour of a more diffuse account in which content is at-issue at a certain point of the discourse just in case it is on the RF of the discourse at that time. Content that is AI becomes NAI when knocked off the RF; content that is NAI can become AI if targeted through discourse subordination, as happens to Wesley's presupposition in $(5 \mathrm{~b} / \mathrm{c})$. The resulting model takes into account semantic and syntactic features of the sentences, as those can play an integral role in determining how discourse is structured, but being at-issue is ultimately a discourse level notion. And while our SDRT discourse semantics exploits public commitments to analyze the contents of conversations so that each speaker commits to what she has asserted (Asher \& Venant 2015), a speaker cannot force her interlocutor to commit to a proposition $p$ just by using a grammatical device. Acceptance, let alone a mutual commitment to content, requires explicit moves by the interlocutor, in line with the ideas in Clark 1992.

\section{The AI/NAI distinction and conversational goals}

While we have argued that how an expression contributes content in the overall discourse structure captures data marshalled in favor of the AI/NAI distinction, we have not denied the observation that motivated syntactic/semantic accounts in the first place-namely, that NAI constructions make wonderful tools for forcing their content into a discourse-secondary role. In this section, we explore the question of why we should have such tools in the first place. It is evident that they are useful for adding background information about an entity under discussion or new material that a speaker finds helpful but less important. Equally important, however, is that they can be used to sneak in controversial information that is central to a speaker's conversational goals. In the remainder of this paper, we argue that a more general account of discourse structure not only yields a superior model of (N)AI content, but also a broader understanding of the usefulness of NAI devices.

13 As suggested by Paul Portner, p.c. 
Shapes of conversation

Consider again the exchange in (5) from the film The Princess Bride. (5) is an example of a non-cooperative conversation in the sense of Asher \& Lascarides 2013, so speaker intentions need not be mirrored by the discourse structure, and the discourse connections that interpreters infer need not adhere to a speaker's intentions as is evident in political debates and other examples (cf. Lepore \& Stone 2015). ${ }^{14}$ In fact, in a strategic conversation in which interlocutors have different discourse goals, NAI content can be more indicative of a speaker's discourse goals (or the issue/question that the speaker aims to discuss) than AI content, contrary to the assumption made in Roberts 2012 inter alia that there is a direct relationship between discourse goals and AI content.

This complicated relationship between (N)AI content and discourse goals makes NAI content a strategically useful device for shifting the issue/question under discussion and, consequently, what counts as AI and NAI content, as we explain below. Importantly, these shifts can go beyond short challenges of NAI content or NAIcentered interludes aimed at clarifying or justifying NAI content discussed in the QUD literature, (e.g., Potts 2012; Roberts 2012; Ginzburg 2012).

Following Asher et al. (2016), we model a conversational goal as a subset of all possible conversations in the sequential game space of all possible discourse moves. The goal of making a conversation coherent, for example, is modelled as the set of all coherent strings of discourse moves. Not angering one's interlocutor might be another goal, denoting a different set of strings. Exogenously given decision problems or conversations aimed at answering a particular question are also instances of such goals. Goals can be complex, formed from combinations of simpler goals. We'll assume that each participant or player $i$ in a conversational game has a goal or winning set $W_{i n}$, and that a strategy for a player $i$ is a function from a prefix of a conversation to a sequence of moves by $i$. Players adopt strategies to steer the conversation into $\mathrm{Win}_{i}$.

Let's now return to (5) and assign Wesley the following conversational goal: to get clear on Buttercup's feelings for Humperdink. This goal, as is clear in the larger discourse from which (5) is taken, is a part of a strategy to achieve a larger discourse goal: to determine whether Buttercup still loves Wesley (and Wesley alone). An answer from Buttercup regarding her feelings for Humperdink takes Wesley one step closer to his larger goal. Notice however, that neither the larger goal nor the sub-goal is straightforwardly reflected in the AI/NAI content distinction: Buttercup's attitude to the presuppositional content in (5c) is ultimately what interests Wesley.

Given that the presuppositional content is actually what is relevant to Wesley's conversational goal, why does he opt for an NAI device - that is, a device that makes this content less accessible? Why not ask Buttercup directly if she loves

14 We note that this view also follows from SDRT's semantics in terms of public commitments. 
Humperdink? By opting for an NAI device, Wesley conceals his discourse goal, leaving Buttercup less likely to consider this goal when planning her own discourse moves. This sets up the situation for a potentially more honest answer, while a direct question might, for example, lead her to be suspicious about the purpose of the question and might make her more likely to dissimulate her true feelings. Using an NAI device has an additional benefit: precisely because it requires more effort to correct a presupposition, a correction of it is all the more meaningful.

Let's see more precisely how the use of the NAI device is useful to Wesley in achieving his winning condition. If Buttercup corrects the NAI content as she does in (5c), then Wesley can assume that she does not love Humperdink and can then pursue his larger goal of determining whether she still loves him. A further benefit of using an NAI device in this case is that when Buttercup corrects the presuppositional content, she becomes responsible for making it AI. This allows Wesley to pursue his personal conversational goals while continuing to conceal them, at least for a while.

But suppose Buttercup had not questioned the NAI content and had responded only to the AI content with, Yes, he will save me. How would such a response have served Wesley? First of all, her acceptance and public commitment ${ }^{15}$ to the NAI content would allow Wesley to cut his losses and infer that her romantic allegiances had shifted to Humperdink. Another consequence is that the discussion of Buttercup's love for Humperdink would remain NAI in this case. This is advantageous because there is no reason for Wesley to pursue the question if Buttercup does love Humperdink, and because it allows him to avoid a potentially painful topic (Buttercup's feelings for Humperdink) or at least awkward questions from Buttercup such as Who are you and why do you want to know?, which would have been triggered by the use of an AI device to achieve Wesley's conversational goal.

To give another example of the usefulness of NAI moves in conversational strategies, consider (20), taken from a voire-dire examination, where the plaintiff lawyer LP is trying to extract information from an expert witness D about a surgery performed by the defendant Dr. Tzeng. After hours of testimony and questioning of $D$ by $L P$ in which they have already discussed at length the operation the defendant had performed, the plaintiff's lawyer uses several NAI devices to slip a claim that the defendant was guilty of negligence if not malpractice. LP's conversational goals in (20) are arguably quite complex. He probably suspects that even if he extracts a commitment from $D$ that the defendant was negligent during the surgical operation, D will be prompted by the defendant's lawyer to reconsider. But his larger goal is to show that $D$ is not impartial as an expert witness, whose testimony is crafted in

15 A speaker's use of a presupposition should not entail that her addressee commits to its content, but once an addressee responds positively to a structured proposal by building on that structure, she commits herself to the whole of both its content and its structure. A rejection move, by contrast, might target only part of the structured proposal. See the discussion of correction in Section 3.3. 
Shapes of conversation

conjunction with the help of the defendant's lawyer to help the defendant.

(20) a. LP: And we know in addition to that, that Dr. Tzeng tore apart this medial antebrachial cutaneous nerve?

b. D: Correct.

c. LD: Objection

d. The Court: Overruled.

e. D: There was a division of that nerve. I'm not sure I would say "tore apart" would be the word that I would use.

f. LP: Oh, there you go. You're getting a hint from your lawyer over here, so do you want to retract what you're saying?

Once again, we can ask about the usefulness of the NAI device as opposed to an AI device in bringing about LP's winning condition. Using an AI device like Did the defendant not exhibit negligence in tearing apart this medial antebrachial cutaneous nerve? would most likely have simply resulted in a simple denial. But by repeatedly coming back to the nature of the operation, LP has primed $D$ to think he's "heard it all before", and set $D$ up to be inattentive to exact wording. Using several NAI triggers to introduce the damning content would have required $D$ to be attentive and to construct a complicated response in which he denied a commitment to the NAI content. But $D$ was apparently inattentive, and issued a short agreement response, which committed $D$ to the defendant's intentional action of tearing apart the nerve and hence to negligence if not malpractice. $L P$ has used the NAI construction to good effect, and we see here that acceptance of NAI content can be incriminating. The ensuing interactions in $(20 \mathrm{a}-\mathrm{c})$ are a perfect setup for LP to make the move in (20f) and make the jury suspect that $D$ was covering something up. Thus, once again we see the interest of an NAI device in strategic conversation.

\section{Conclusions}

We have presented a discourse based account of the behavior of appositives and in principle other expressions that are ordinarily thought to convey NAI content. We've shown that whether the content conveyed by such constructions is NAI or not depends upon the discourse structure in which these contents are embedded. Further, we've shown that the distinction between NAI and AI content is not a simple binary one but more nuanced and that a discourse based account predicts a much wider range of phenomena than competing accounts based on multidimensional semantics or multiple update mechanisms. Finally, we have shown how a strategic conception of dialogue can shed light on the uses of these constructions in discourse structure. 
Hunter and Asher

\section{References}

Afantenos, Stergos, Eric Kow, Nicholas Asher \& Jérémy Perret. 2015. Discourse parsing for multi-party chat dialogues. In Lluis Marquez, Chris Callison-Burch \& Jian Su (eds.), The 2015 Conference on Empirical Methods in Natural Language Processing (EMNLP), 928-937. Lisbon, Portugal: Association for Computational Linguistics.

Amaral, Patricia, Craige Roberts \& E. Allyn Smith. 2007. Review of the Logic of Conventional Implicatures by Chris Potts. Linguistics and Philosophy 30(6). 707-749. doi:10.1007/s10988-008-9025-2.

AnderBois, Scott, Adrian Brasoveanu \& Robert Henderson. 2015. At-issue proposals and appositive impositions in discourse. Journal of Semantics 32(1). 93-138. doi:10.1093/jos/fft014.

Asher, Nicholas. 1993. Reference to Abstract Objects in Discourse. Kluwer Academic Publishers.

Asher, Nicholas. 2000. Truth conditional discourse semantics for parentheticals. Journal of Semantics 17(1). 31-50. doi:10.1093/jos/17.1.31.

Asher, Nicholas \& Alex Lascarides. 1998. The semantics and pragmatics of presupposition. Journal of Semantics 15(3). 239-299. doi:10.1093/jos/15.3.239.

Asher, Nicholas \& Alex Lascarides. 2003. Logics of Conversation. Cambridge University Press.

Asher, Nicholas \& Alex Lascarides. 2013. Strategic conversation. Semantics and Pragmatics 6(2). 1-62. doi:10.3765/sp.6.2.

Asher, Nicholas \& Soumya Paul. 2013. Infinite games with uncertain moves. In Fabio Mogavero, Aniello Murano \& Moshe Y. Vardi (eds.), The First International Workshop on Strategic Reasoning, 25-32. Rome.

Asher, Nicholas, Soumya Paul \& Antoine Venant. 2016. Message exchange games in strategic conversation. Journal of Philosophical Logic 1-50. doi:10.1007/s10992-016-9402-1.

Asher, Nicholas \& Antoine Venant. 2015. Ok or not ok? In Sarah D’Antonio, Mary Moroney \& Carol Rose Little (eds.), Semantics and Linguistic Theory (SALT) 25, Cornell University Press.

Clark, Herbert. 1992. Arenas of Language Use. Chicago: University of Chicago Press.

Farkas, Donka \& Kim B. Bruce. 2010. On reacting to assertions and polar questions. Journal of Semantics 27(1). 81-118. doi:10.1093/jos/ffp010.

Ginzburg, Jonathan. 2012. The Interactive Stance: Meaning for Conversation. Oxford University Press.

Jasinskaja, Katja. 2016. Salience and (not-)at-issue status of subordinate clauses. In Sinn und Bedeutung 21, Presentation. 
Shapes of conversation

Koev, Todor. 2012. On the information status of appositive relative clauses. In Maria Aloni, Vadim Kimmelman, Floris Roelofsen, Galit Sassoon, Katrin Schulz \& Matthijs Westera (eds.), The Amsterdam Colloquium 18 (Revised and Selected Papers), 401-410. Springer.

Lepore, Ernest \& Matthew Stone. 2015. Imagination and Convention: Distinguishing Grammar and Inference in Language. Oxford University Press.

Muller, Philippe, Stergos Afantenos, Pascal Denis \& Nicholas Asher. 2012. Constrained decoding for text-level discourse parsing. In Martin Kay \& Christian Boitet (eds.), The International Conference on Computational Linguistics (COLING), 1883-1900. Mumbai, India.

Murray, Sarah. 2011. A Hamblin semantics for evidentials. In Ed Cormany, Satoshi Ito \& David Lutz (eds.), Semantics and Linguistic Theory (SALT) 19, 324-341.

Perret, Jérémy, Stergos Afantenos, Nicholas Asher \& Mathieu Morey. 2016. Integer linear programming for discourse parsing. In Kevin Knight, Ani Nenkova \& Owen Rambow (eds.), The 2016 North American Chapter of the Association for Computational Linguistics, 99-109. San Diego, CA, USA: Association for Computational Linguistics.

Potts, Christopher. 2005. The Logic of Conventional Implicatures. Oxford University Press Oxford.

Potts, Christopher. 2012. Conventional implicature and expressive content. In Claudia Maienborn, Klaus von Heusinger \& Paul Portner (eds.), Semantics: an international handbook of natural language meaning, 2516-2536. Berlin: Mouton de Gruyter.

Reese, Brian. 2007. Bias in questions: The University of Texas at Austin PhD dissertation.

Roberts, Craige. 2012. Information structure in discourse: Towards an integrated formal theory of pragmatics. Semantics and Pragmatics 5(6). 1-69. doi:10.3765/sp.5.6.

Simons, Mandy, Judith Tonhauser, David Beaver \& Craige Roberts. 2010. What projects and why? In Nan Li \& David Lutz (eds.), Semantics and Linguistics Theory (SALT) 20, 309-327.

Syrett, Kristen \& Todor Koev. 2015. Experimental evidence for the truth conditional contribution and shifting information status of appositives. Journal of Semantics 32(3). 525-577. doi:10.1093/jos/ffu007.

Wilson, Deirdre. 1975. Presuppositions and Non Truth Conditional Semantics. New York: Academic Press. 
Hunter and Asher

Julie Hunter

IRIT, Université Paul Sabatier

118 route de Narbonne

31062 Toulouse, France

juliehunter@gmail.com
Nicholas Asher

IRIT, Université Paul Sabatier

118 route de Narbonne

31062 Toulouse, France

asher@irit.fr 